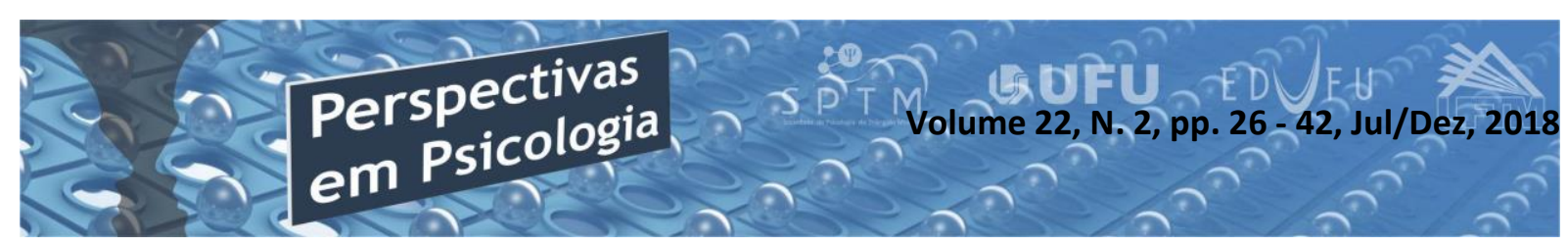

\title{
ALTERAÇÕES DE SAÚDE MENTAL EM PESSOAS QUE VIVEM COM HIV/AIDS
}

\author{
Aurélio Goulart Rodovalho \\ Roselma Lucchese \\ Moises Fernandes Lemos \\ Henrique Senna Diniz-Pinto \\ Lucas Barros Araújo \\ Patrícia Mendes Barros \\ (Universidade Federal de Goiás, Regional Catalão)
}

\begin{abstract}
Resumo
Objetivou-se com esse estudo estimar a prevalência de depressão entre pessoas que vivam com HIV/Aids e verificar a correlação entre instrumentos de rastreio de depressão e de Transtorno Mental Comum. Trata-se de um estudo transversal, de natureza quantitativa realizado com uma amostra composta por 83 indivíduos que vivem com HIV/Aids em atendimento em um serviço de referência no Brasil Central entre os anos de 2017 e 2018. Os resultados do presente estudo apontaram uma prevalência de 53\% de depressão e associação positiva com ansiedade, distúrbio do sono, ideação suicida e baixa renda. Os escores dos instrumentos para rastreio de depressão e de Transtorno Mental Comum se correlacionaram.
\end{abstract}

Palavras chave: HIV; AIDS; Saúde Mental; Depressão.

\section{Abstract}

\section{Mental health changes in people living with HIV / AIDS}

The objective of this study was to estimate the prevalence of depression among people living with HIV / AIDS and to verify the correlation between screening instruments for depression of Common Mental Disorder. It is a cross-sectional study of a quantitative nature carried out with a sample composed of 83 individuals living with HIV / AIDS in a referral service in Central Brazil between 2016 and 2018. The results of the present study indicated a high prevalence of $53 \%$ of depression and positive association with anxiety, sleep disorder, suicidal ideation and low income. The scores of the instruments for evaluation of depression and Common Mental Disorder correlated.

Keywords: HIV; AIDS; Mental Health; Depression. 


\section{Introdução}

A infecção pelo Vírus da Imunodeficiência Humana (HIV) tornou-se problema de saúde pública em escala global. Estima-se que 37 milhões de pessoas vivam com HIV/Aids (PVHA) (Onusida, 2015). Em um processo saúde doença marcado por um período assintomático, a infecção provoca a deficiência do sistema imunológico seguido por suscetibilidade à doenças oportunistas e neoplasias. Contudo, o desenvolvimento de uma terapia antirretroviral (TARV) alterou o prognóstico da epidemia com efetivo controle da atividade viral, recomposição da função imunológica e considerável redução na taxa de mortalidade (Tancredi \& Waldman, 2014).

Entretanto, paralelo ao avanço das políticas de tratamento, a atenção tem focado na melhoria da qualidade de vida de PVHA, já que os impactos psicossociais se apresentam como fatores de riscos para problemas crônicos (Fernandez \& Torvisco, 2014; Egbe et al., 2017). Nesse sentido, evidências científicas indicaram que eventos de estresse, depressão, ansiedade e ideação suicida impulsionaram as comorbidades com efeitos psicopatológicos e têm sido comumente associados à PVHA
(Mola et al., 2016; Aguillar, Maycott, Iglesias, Estrada, \& Téran, 2017).

A compreensão do impacto psicossocial da infecção na vida de PVHA tem sido instrumento de estudo desde o início da epidemia (Fernandez \& Torvisco, 2014; Reis et al., 2017). Nessa perspectiva, a totalidade dos autores associaram a presença da depressão ao nível de estigma, baixo suporte social, isolamento, baixa autoestima e ainda como resultado da própria atividade viral (Passos \& Souza, 2015; Schlebusch \& Govender, 2015; Egbe et al., 2017; Reis et al., 2017; Wang et al., 2018). Esses elementos, de um modo geral, prejudicam o tratamento, pois são fortemente associados à baixa adesão a TARV (Sin \& DiMatteo, 2014).

Em tese, PVHA têm maiores chances de apresentar sintomas de depressão, desse modo, o conhecimento dessa ocorrência garantirá o diagnóstico imediato da condição mental e priorizará as intervenções de promoção da saúde mental e redução de danos à saúde das PVHA. Os objetivos do presente estudo foram: estimar a prevalência de depressão entre pessoas que vivam com HIV/Aids; verificar a correlação entre instrumentos de rastreio de depressão de Transtorno Mental Comum. 
Trata-se de um estudo transversal, de natureza quantitativa. $\mathrm{O}$ estudo foi desenvolvido em um Serviço de Atendimento Especializado (SAE) para PVHA localizado no Brasil Central.

\section{População e Amostra}

A população do estudo foram todos os pacientes acompanhados pelo SAE. Para a amostra atendeu-se os critérios de inclusão que foram: PVHA com mais de 13 anos em tratamento $\geq$ seis meses; ambos os gêneros. Os critérios de exclusão foram: gestantes; indivíduos que não apresentaram capacidade cognitiva suficiente para responder aos instrumentos e os que não se apresentaram no SAE durante o período de coleta de dados. A amostragem do estudo se deu por conveniência.

\section{Coleta de Dados}

Para validação dos métodos adotados, inicialmente aplicou-se um teste piloto com três indivíduos que estavam em tratamento na unidade, entretanto os resultados não foram utilizados na amostra final. Esse momento representou-se de extrema relevância pelo treinamento dos pesquisadores de campo e, de fomento de estratégias operacionais da pesquisa.
Os pacientes foram convidados a participar do estudo durante o período em que se apresentavam para as consultas ou a retirada das medicações. Todos os indivíduos que aceitaram participar do estudo assinaram um Termo de Consentimento Livre e Esclarecido. A coleta de dados ocorreu entre janeiro de 2017 e maio de 2018. O método de coleta de dados foi a entrevista face-a-face em local privativo no próprio SAE por profissionais que compunham a equipe do serviço.

\section{Instrumentos Aplicados}

Todos os instrumentos utilizados na coleta de dados foram transcritos para uma plataforma online digital através da ferramenta Google Formulários abrigada pelo Google Drive, onde os dados são inseridos e armazenado nas nuvens. Isso possibilitou a exportação dos resultados automaticamente em formato Excel. Um estudo demostrou a eficácia desse recurso tecnológico na coleta de dados estatísticos em áreas da saúde, o que facilita o registro do paciente e aperfeiçoa o registro e intercâmbio das informações (Lopes, Gracitelli \& Moura, 2018).

A primeira etapa da coleta de dados ocorreu com a aplicação do questionário sóciodemografico. No que se baseou em 
estudos anteriores que avaliaram a frequência de efeitos psicossociais em PVHA (Souza Junior, Szwarcwald \& Castilho, 2011; Gaspar et al., 2011; Passos \& Souza, 2015).

Quanto à saúde mental foram avaliados os níveis de depressão, ansiedade, ideação suicida, presença de transtorno mental comum, qualidade do sono e utilização de substâncias psicoativas.

Para avaliar a presença de sintomas depressivos utilizou-se o Inventário de Depressão de Beck (BDI). Trata-se de um questionário composto por 21 itens de múltipla escolha (Beck, Ward, Mendelson, Mock, \& Erbaugh, 1961) validado na versão português (Cunha, 2001). O escore de depressão foi obtido através da somatória da pontuação do inventário e classificado em ausência de sintomas depressivos ou depressão mínima (escore com pontuação de 0 a 11), depressão leve (escore de 12 a 19 pontos), depressão moderada (escore de 20 a 35 pontos) e depressão grave (escore de 36 a 63 pontos). O BDI é um instrumento comumente utilizado para identificar a intensidade de episódios depressivos em PVHA (Barata et al., 2013; Silveira, 2012).

Para determinar os níveis de ansiedade utilizou-se o Inventário de Ansiedade de Beck (BAI). Também, composto por 21 itens, o escore foi obtido pela somatória dos itens e classificado em ausência de sintomas de ansiedade (escore com pontuação de 0 a 10), ansiedade leve (escore de 11 a 19 pontos), ansiedade moderada (escore de 20 a 30 pontos) e ansiedade grave (escore de 31 a 63 pontos). Trata-se de um instrumento largamente utilizado para medir a severidade da ansiedade do indivíduo (Junqueira, Ward, Mendelson, Mock, \& Erbaugh, 2008).

Para Ideação suicida foi utilizado o Inventário de Ideação Suicida de Beck (BSI). As pontuações foram somadas para determinação do escore e classificados em ausência de ideação suicida (escore igual a zero) e presença de ideação suicida (qualquer valor superior a zero) (Baldo, 2017).

Para o rastreamento de TMC aplicou-se o instrumento Self-Reporting Questionnaire (SRQ-20). O instrumento foi desenvolvido pela Organização Mundial de Saúde (OMS), fundamentada pela preocupação com os impactos que os problemas de saúde mental poderiam apresentar e validado na língua portuguesa em 1986 (Mari \& Williams, 1986). O instrumento se mostrou eficaz para identificação de transtorno mental comum entre PVHA (Nakimuli-Mpungu et al., 2012). O escore foi obtido através da somatória da pontuação e os resultados foram classificados considerando o ponto de corte igual a sete. Dessa forma, escores $\leq 6$ foram classificados como ausência de TMC e para escore $\geq 7$ presença de TMC. 


\section{Variáveis}

Variável dependente: Depressão. Para a classificação dos resultados e posterior análise foram criadas duas categorias: não apresenta sintomas de depressão vs. apresenta sintomas de depressão (depressão leve, moderada e grave).

Variáveis independentes: Gênero (Masculino vs. Feminino); Escolaridade ( $<8$ anos vs. $\geq 8$ anos); Renda per capta, obtida através da divisão da renda total da família por moradores na residência (renda per capta $<1.500$ reais vs. renda per capta $\geq 1.500$ reais); Estado civil (vive com companhia vs. vivem sem companhia); Filhos (sim vs. não); Cansaço, questionou se o indivíduo sente-se cansado (sim vs. não); Fadiga, questionou se o indivíduo apresenta fadiga (sim vs. não); Tontura, questionou se o indivíduo apresenta tontura ( $\operatorname{sim}$ vs. não); Ansiedade, baseada no instrumento BAI foram criadas duas categorias: sem sintomas vs. com sintomas (ansiedade leve, moderada e grave); Sono, questionou-se: "na sua opinião a qualidade de seu sono foi bom vs. ruim); Suicídio (apresenta ideação suicida vs. não apresenta); Uso de hipnóticos/sedativos, questionou-se "faz uso de algum remédio para dormir" (sim vs. não); Uso da combinação antirretroviral Tenofovir (TDF) 300mg + Fumarato de Lamivudina (3TC) 300mg e Dolutegravir (DTG) 50mg, nesse caso a pesquisa da indicação do medicamento foi feito por revisão dos prontuários (sim vs. não).

\section{Análise Estatística}

Os dados coletados em instrumento digital geraram planilha que constitui o banco de dados. O processo de análise ocorreu em programa Data Analysis and Statiscal Software (STATA) versão 14,0. As variáveis categóricas foram descritas com números absolutos, frequência, intervalo de confiança 95\% (IC:95\%); as variáveis contínuas descritas por média, IC:95\% e desvio padrão (DS). Nas análises bivariadas e múltiplas aplicou-se regressão logística com medida de efeito Odds Ratio $(\mathrm{OR})$, e o teste quiquadrado para as diferenças de proporção, considerou-se significantes $\mathrm{p}<0,05$ na análise múltipla. Também na análise múltipla avaliou-se a qualidade de ajuste do modelo pelo teste Hosmer e Lemeshow. Para a correlação entre os escores de BDI e TMC realizou-se regressão linear.

\section{Resultados}


A amostra do estudo constituiu-se de 83 PVHA. Houve perda de três por inconsistência do questionário. A média de idade foi de 41,3 anos (IC95\%, SD=11,17), com predomínio de homens $(64 \%)$ e baixa escolaridade ( $47 \%$ estudaram menos de oito anos). Dos entrevistados $47 \%$ afirmaram ter um companheiro (a), 58\% têm filhos, 53\%

Tabela 1 Análise bivariada entre a variável dependente e fatores associados à depressão em

PVHA. Brasil Central, 2018

\begin{tabular}{|c|c|c|c|c|}
\hline & \multicolumn{2}{|c|}{ Depressão } & \multirow{2}{*}{$\frac{\mathrm{OR}^{*}}{(\mathrm{IC} 95 \%)^{* *}}$} & \multirow[t]{2}{*}{$\mathrm{P}$} \\
\hline & $\begin{array}{c}\text { Não } \\
\text { N (\%) }\end{array}$ & $\begin{array}{c}\mathrm{Sim} \\
\mathrm{N}(\%)\end{array}$ & & \\
\hline \multicolumn{5}{|l|}{ Gênero } \\
\hline Masculino & $30(56,6)$ & $23(43,4)$ & 1 & \\
\hline Feminino & $09(30,0)$ & $21(70,0)$ & $\begin{array}{c}3,043(1,175- \\
7,879)\end{array}$ & 0,022 \\
\hline $\begin{array}{l}\text { Escolaridade } \\
\geq 8 \text { anos de } \\
\text { estudo }\end{array}$ & $25(64,10)$ & $19(43,18)$ & 1 & \\
\hline $\begin{array}{l}<8 \text { anos de } \\
\text { estudo }\end{array}$ & $14(35,90)$ & $25(56,82)$ & $\begin{array}{c}2.349(0,969- \\
5,695)\end{array}$ & 0,059 \\
\hline $\begin{array}{l}\text { Renda } \text { /Capta } \\
\geq 1,500 \text { reais } \\
\text { por pessoa }\end{array}$ & $14(35,90)$ & $25(64,10)$ & 1 & \\
\hline $\begin{array}{l}<1,500 \text { reais por } \\
\text { pessoa }\end{array}$ & $08(18,18)$ & $36(81,82)$ & $\begin{array}{c}2,52(0,920- \\
6,901)\end{array}$ & 0,072 \\
\hline Estado Civil & & & & \\
\hline $\begin{array}{l}\text { Vive com } \\
\text { companhia }\end{array}$ & $16(41,03)$ & $23(58,97)$ & 1 & \\
\hline $\begin{array}{l}\text { Vive sem } \\
\text { companhia }\end{array}$ & $19(43,18)$ & $25(56,82)$ & 0,915 & 0,843 \\
\hline Filhos & & & & \\
\hline Não & $24(68,57)$ & $11(31,43)$ & 1 & \\
\hline Sim & $15(31,25)$ & $33(68,75)$ & $\begin{array}{c}4,8(1,876- \\
12,276)\end{array}$ & 0,001 \\
\hline Cansaço & & & & \\
\hline Não & $26(60,47)$ & $17(39,53)$ & 1 & \\
\hline
\end{tabular}

recebem menos de DOIs salários mínimos por mês e $78 \%$ tem alguma religião.

Referente às condições de saúde mental, a prevalência de depressão na amostra estudada foi de 53\% ( $\mathrm{n}=44$, IC95\%). Na tabela 1 apresentou-se a análise bivariada e fatores associados à depressão. 
$\operatorname{Sim}$

$13(32,5)$

$27(67,5)$

Fadiga

Não

Sim

$34(59,65)$

$04(16,0)$

Tontura

Não

$30(58,82)$

Sim

$09(28,13)$

Ansiedade

Sem Sintomas

$33(84,62)$

Apresenta

$06(13,64)$

Sintomas

Qualidade do sono

Não

Sim

$30(66,67)$

$09(23,68)$

Suicídio

Sem ideação

Ideação

$37(52,11)$

$02(16,67)$

Risco Hipnótico

Sedativos

Baixo risco

Risco Moderado

$39(50,0)$

$0(0,0)$

TNF/3TC+ DTG

Não

Sim
$34(43,59)$

$05(100,0)$
$23(40,35)$

$21(84,0)$

$21(41,18)$

$23(71,88)$

$06(15,38)$

$38(56,36)$

$15(33,33)$

$29(76,32)$

$34(47,89)$

$10(83,33)$

$39(50,0)$

$05(100,0)$

$1,128(1,026-$

$1,281)$

$44(56,41)$

$0(0,0)$

1

$6,208(2,046-$

$18,831)$

$$
-118,450)
$$

1

$6,444(2,439-$

17,022)

1

$5,441(1,111-$

$26,628)$

\section{1}

1
$3,176(1,290-$

0,012

$7,819)$

0,001

0,000

0,037

0,030

$0,872(0,755-\quad 0,014$

*Odds ratio (OD). ** Intervalo de Confiança 95\%

$\mathrm{Na}$ análise bivariada o estudo demonstrou associação entre a variável dependente ter sintomas de depressão e variáveis independentes sociodemográficas (gênero, escolaridade, renda e filhos). Em destaque, os resultados da análise bivariada indicaram que ter filhos representou aproximadamente cinco vezes mais chances de desenvolver depressão. Além disso, associou-se também com sintomas como cansaço, fadiga, tontura, ansiedade, qualidade do sono auto referida, uso de hipnóticos/sedativos e uso da combinação de antirretrovirais TNF/3TC + DTG.

Pela análise bivariada foram selecionadas as variáveis que apresentaram 
valor de $\mathrm{p}<0,10$. Na tabela 2 demonstrou-se os resultados da análise múltipla por regressão logística. As variáveis Risco Hipnóticos/Sedativos e uso de antirretrovirais $\mathrm{TNF} / 3 \mathrm{TC}+\mathrm{DTG}$ apresentaram $\mathrm{p}<0,10$, contudo não foram selecionadas para análise múltipla por tererm apresentado em um dos campos da tabela o valor zero, comprometendo-se a qualidade da análise estatística.

Tabela 2 Análise múltipla entre variável dependente e fatores associados à depressão em PVHA. Brasil Central, 2018

\begin{tabular}{|c|c|c|}
\hline Variáveis & ORaj* $^{*}(\mathrm{IC} 95 \%)^{* *}$ & $p$-value \\
\hline \multicolumn{3}{|l|}{ Depressão } \\
\hline Gênero Feminino & $0,487(0,0748-3,163)$ & 0,451 \\
\hline$<8$ anos de Estudo & $1,346(0,199-9,099)$ & 0,760 \\
\hline$<1,500$ Reais de renda per capta & $7,339(1.057->10)$ & 0,044 \\
\hline Tem Filhos & $4,284(0,531->10)$ & 0,172 \\
\hline Apresenta Cansaço & $1,908(0,319->10)$ & 0,479 \\
\hline Apresenta Fadiga & $0,903(0,114-7,576)$ & 0,946 \\
\hline Apresenta Tontura & $1,370(0,173->10)$ & 0,765 \\
\hline Apresenta sintomas de ansiedade & $10,523(4,980->10$ & 0,000 \\
\hline Dorme mal & $5,987(1,129->10)$ & 0,035 \\
\hline Ideação Suicida & $22,104(1,173->10)$ & 0,039 \\
\hline
\end{tabular}

A análise múltipla apresentou associação positiva para as variáveis $<1.500$ reais de renda per capta, apresenta sintomas de ansiedade, dorme mal e ideação suicida. 
Gráfico 1: Análise de regressão linear entre variáveis contínuas do escore do BDI vs. escore do SRQ-20 para transtorno mental comum, Brasil Central, 2018

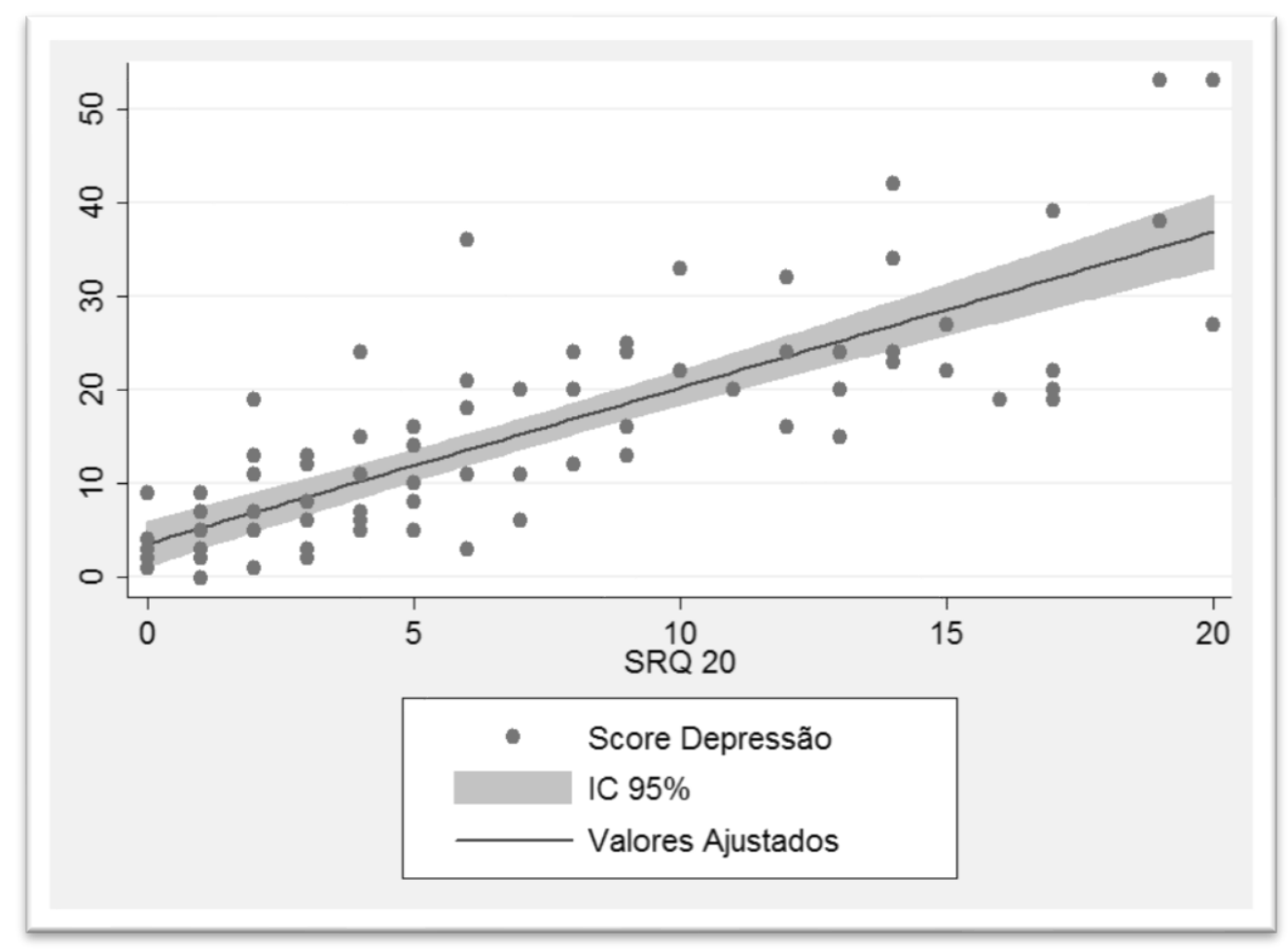

Observa-se no Gráfico 1 a análise linear estão demonstrados no associação positiva entre os escores do BDI Quadro 1.

com SRQ20. Os valores numéricos da

Quadro 1 Regressão linear para os escores totais de BDI (Depressão) e do escore do instrumento SRQ-20 (Transtorno Mental Comum). Brasil Central, 2018

\begin{tabular}{|lccc|}
\hline DEPRESSÂO & Coef. de Correlação & p-value & IC 95\%* \\
TMC & 1.662 & 0,000 & $1.400-1.924$ \\
\hline
\end{tabular}

* Intervalo de Confiança 95\% 
A análise de correlação linear entre os escores BDI e SRQ20 apontou que a cada elevação de um ponto no SRQ-20 aumentase 1,662 no escore do BDI, confirmando-se

\section{Discussão}

Os resultados do presente estudo contribuíram para o melhor conhecimento do tema uma vez que aproximadamente metade das PVHA que compuseram a amostra apresentaram sintomas de depressão. Esse dado foi associado com outras alterações de saúde mental como ansiedade, transtorno mental comum, ideação suicida e distúrbio do sono.

Este estudo apresentou limitações quanto ao desenho transversal, pois impossibilitou identificar as relações causais entre variáveis pesquisadas. Admite-se como viés de estudo o da memória, ao considerar que as respostas aos instrumentos, embora fossem questões referentes aos últimos 30 dias, podem ter ocorrido algumas omissões ou esquecimentos.

Observou-se prevalência $53 \%$ de sintomas depressivos pelo escore do BDI entre PVHA. Esses achados foram superiores do que outros estudos, que apresentaram prevalência que variaram de 30 a 40\% (Souza Junior et al., 2011; Egbe et al., 2017; Reis et al., 2017; Wang et al., então a correlação positiva entre o resultado dos DOIs instrumentos.

2018). Uma das justificativas foi que no atual estudo considerou-se para a prevalência os escores do BDI para os graus de depressão leve, moderado e grave, por entendimento de que qualquer sintoma depressivo exigirá atenção das equipes multidisciplinares que atende a PVHA. Esses fatos mostram a necessidade de compreensão das condições que favorecem o adoecimento por depressão entre a população estudada.

Estudos anteriores sugeriram que fatores sociais e psicológicos como exclusão social, estigma e dificuldade em manter relações afetivo-sexuais, podem ser os responsáveis pelo aumento da prevalência de depressão entre PVHA. Por outro lado, depressão também foi associada a problemas no sistema imunológico, principalmente redução do número de linfócitos $\mathrm{CD} 8$, o que permite a progressão da atividade viral (Evans et al., 1997; Reis et al., 2017).

O presente estudo apresentou associação positiva entre renda per capta e depressão, em que pessoas com renda $<1.500$ reais têm 7,339 mais chances de desenvolver depressão. Achados semelhantes foram encontrados (Passos \& 
Souza, 2015; Gaspar et al., 2011). Estudos anteriores mostraram que, por se tratar de populações que vivem em países em desenvolvimento, o nível socioeconômico é parcialmente definido como resultado da qualificação educacional e a situação profissional. Essas condições estão diretamente ligadas ao status de saúde, competência funcional e qualidade de vida de um modo geral (Souza Junior et al., 2011; Passos \& Souza, 2015; Caliari, Teles, Reis, \& Gir, 2017). Assim, o indicador de condições socioeconômicas considerou as necessidades materiais, como a possibilidade de manter condições satisfatórias de moradia, saúde e nutrição, e ainda agiu como parâmetro de bem-estar social (Passos \& Souza, 2015), o que explica a associação entre baixa renda e depressão.

Como esperado, os resultados mostraram que entre a ansiedade $\mathrm{e}$ depressão houve associação positiva. Indivíduos com sintomas de ansiedade apresentaram 30,52 vezes mais chances de desenvolver depressão quando comparado aos que não tinham sintomas de ansiedade. Esses dados foram consistentes com outros estudos realizados com PVHA (Passos \& Souza, 2015; Liu, 2017). Além disso, estudos apontaram que depressão e ansiedade são mais frequentes entre PVHA do que na população em geral (Souza Junior et al., 2011), uma vez que aceitar uma condição crônica, conviver com o medo da morte, o estigma e ainda suportar os efeitos colaterais produzidos pelo uso da medicação provocou sofrimento mental intenso. Esses dados são de extrema importância, uma vez que efeitos psicopatológicos podem influenciar diretamente o sistema imunológico do indivíduo levando a progressão da carga viral e, por consequência, prejuízo da reconstituição imunológica (Sin \& DiMatteo, 2014; Passos \& Souza, 2015).

Nesta perspectiva, há ainda a preocupação que permeia sobre os problemas psicológicos e a adesão ao tratamento. Evidências científicas apontam que indivíduos com sintomas de depressão e ansiedade foram mais vulneráveis a comportamentos suicidas e ainda apresentaram uma tendência maior a abandonar o tratamento (Sin \& DiMatteo, 2014). Desse modo, estudos sugeriram o fortalecimento de ações de triagem e acompanhamento aos indivíduos que apresentam alterações nas avaliações de saúde mental (Aguillar et al., 2017; Egbe et al., 2017; Reis et al., 2017).

Observou-se que os indivíduos que classificaram seu sono como ruim apresentaram seis vezes mais chances de desenvolver depressão. Distúrbios do sono já são esperados em indivíduos com 
quadros de sofrimento mental. Entretanto, PVHA podem também desenvolver alterações no sono em decorrência da neurotoxicidade pelo uso de TARV e pela própria atividade viral no sistema nervoso central (Junqueira et al., 2008; Treisman \& Soudry, 2016).

A associação entre depressão e alterações no sono igualmente foi encontrada em outros estudos. Seus resultados mostraram que os indivíduos mais afetados apresentaram mais ocorrência de distúrbios noturnos diários. As perturbações do sono provocam situações de tristeza, impaciência, hiperatividade, isolamento e perda de ânimo e energia, os quais influenciam de forma negativa na qualidade de vida. Esse sistema pode funcionar como um ciclo, em que o aumento da depressão provoca alterações no sono e estas, por sua vez aumenta a chance de sintomas depressivos (Junqueira et al., 2008; Morais et al., 2017).

Outro ponto relevante do estudo diz respeito à ideação suicida. PVHA e suicídio representam sérios problemas de saúde pública, em especial em países em desenvolvimento (Schlebusch \& Govender, 2015). Assim como a depressão, a ideação suicida é maior entre PVHA do que na população em geral (Wang et al., 2018). Neste contexto, os resultados do presente estudo apresentaram associação entre ideação suicida e depressão. Observou-se que indivíduos com depressão apresentaram 22,1 vezes mais chances de ideação suicida, quando comparado com quem não tinha sintomas depressivos. Esses dados corroboram com a produção do conhecimento, a qual apresenta que a prevalência de ideação suicida varia entre 25 e $35 \%$ após o diagnóstico de HIV. Dados que sugeriram que a presença de fatores psicossociais como ansiedade e depressão provocou efeito negativo que pode intensificar o estresse psicológico de PVHA e levar a ideação suicida, ao considerarem o suicídio como uma opção para encerrar o sofrimento. (Wang et al., 2018). Contudo, embora a depressão seja um fator preponderante para a ideação suicida, evidências científicas mostraram que o sinergismo de fatores psicossociais provocou um aumento da ideação suicida (Egbe et al., 2017; Wang et al., 2018).

Embora as variáveis "uso de hipnóticos/sedativos" e "uso da combinação antirretroviral TNF/3TC + DTG" não foram para análise múltipla, houve associação positiva na análise bivariada. Evidências científicas mostraram que determinados antirretrovirais, em especial os que compõem a classe dos inibidores de transcriptase reversa não análogos de nucleosídeos (NNRTI), foram comumente associados a efeitos colaterais como alucinações e alterações no padrão do sono (Rachid, 2008; Ahmadi, Khalili, Abbasian, 
\& Ghaeli, 2017). Diante disso, um estudo mostrou que a prescrição de hipnóticosedativos foi uma estratégia eficaz para prevenir efeitos colaterais neuropsiquiátricos, já que essas drogas exerceram um efeito calmante ao reduzirem a excitação (Ahmadi et al., 2017).

Próximos a segunda década do século XXI, existem mais de 16 milhões de PVHA em uso da TARV de primeira escolha baseado em NNRTI. Estudos anteriores mostraram que uma droga que compõe a classe dos NNRTI, o Efavirenz (EFV), foi associada também a outros efeitos psicopatológicos como depressão, alterações de humor e ideação suicida (Ahmadi et al., 2017; Mugusi et al., 2018; Vitoria et al., 2018). Entretanto, para PVHA que apresentem sintomas depressivos ou ideação suicida sugere-se substituir o EFV por DTG (Brasil, 2018). Essa pode ser uma possível explicação para a associação positiva entre o esquema TNF/3TC + DTG e depressão.

A análise mostrou que os instrumentos BDI e SRQ20 se corroboram, no sentido de que as elevações do escore do BDI são acompanhadas pelo aumento no escore do SRQ-20. Essa correlação positiva foi apontada também em outros estudos que compararam a validação das escalas em grupos específicos. Nesses casos, observouse a compatibilidade dos instrumentos
(Marcolino \& Iacoponi, 2003; Salle, Rocha, Rocha, Nunes, \& Chaves, 2012; Mola et al., 2016).

\section{Conclusão}

Pode-se considerar a Aids como uma doença que requer atenção, responsabilidades de assistência e cuidados específicos, seja das pessoas acometidas ou daquelas envolvidas diretamente com o seu tratamento. Exige uma rede profissional multidisciplinar capacitada e apta a oferecer ao paciente as condições necessárias para o enfrentamento do processo de morte que, embora com evolução crônica, requer muitas mudanças em seu padrão de vida.

A prevalência de depressão em PVHA foi alta em relação aos outros estudos com a mesma população e, houve associações com baixa renda, ansiedade, distúrbio do sono e ideação suicida. Em geral, PVHA ainda são mais acometidas por psicopatologias do que a população em geral. Este estudo contribuiu com informações que priorizam o fortalecimento de ações de assistência a saúde mental em unidades de referência para HIV/Aids. Ademais, a utilização de instrumentos que rastreiem depressão no fluxo de atendimentos às PVHA, se mostrou eficiente para o BDI e SRQ-20, e pode transformar o processo de acolhimento nos 
serviços especializados mais assertivo, sobretudo para a promoção da saúde mental e, redução de danos de uma doença que atingiu o status de crônica nesse século.

\section{Referências}

Aguilar C. A., Maycott R. C., Iglesias, M. C., Estrada E.R, \& Terán E. R. (2017). Assessment of depression, anxiety, hopelessness and suicidal risk in HIV+ inpatients. Salud mental, 40(1). DOI.org/10.17711/SM.0185-3325.2017.004

Ahmadi M., Khalili H., Abbasian L., \& Ghaeli P. (2017). Effect of Valerian in Preventing Neuropsychiatric Adverse Effects of Efavirenz in HIV-Positive Patients: A Pilot Randomized, Placebo-Controlled Clinical Trial. Ann Pharmacother, 51(6), 457-464. Recuperado de https://www.ncbi.nlm.nih.gov/pubmed/28478716. DOI: $10.1177 / 1060028017696105$

Baldo V. H. F. S., Junior J. C. T., Rodrigues F. T., Cardoso M. P., Gjorup A. L. T., Dahia M. M. B., Reis J. O. S., Motta R. N., Brasil F. V., Kalil R. S., Soares P. K., \& Schmidt S. L. (2017). Avaliação do risco de suicídio em pacientes HIV positivos em acompanhamento ambulatorial no Hospital Universitário Gaffrée e Guinle. Cad Bras Med, 65(4)

Barata A., Malouf J., Gutierrez M., Mateo G. M., Sambeat M. A., Gich I., Cadafalch, J., Wulff J., \& Domingo P. (2013). Psychopathology and psychosocial adjustament in patients with HIV-associated lipodystroph. The Brazilian Journal of Infectious Diseases, 4

Beck A. T., Ward C. H., Mendelson M, Mock J, \& Erbaugh J. (1961); An inventory for measuring depression. Arch Gen Psychiatry, 41, 561-71. Recuperado de https://www.ncbi.nlm.nih.gov/pubmed/13688369

Brasil. (2018). Nota informática $n^{\text {o }}$ 03/2018. Recuperado de https://sei.saude.gov.br/sei/controlador.php?acao=documento imprimir web\&acao orige $\underline{m}=$ arvore_visualizar\&id_documento $=3764923 \&$ infra_sistema $=1000001$

Caliari J. S., Teles S. A., Reis R. K., \& Gir E. (2017). Factors related to the perceived stigmatization of people living with HIV. Rev Esc Enferm USP, 0. Recuperado de http://www.scielo.br/pdf/reeusp/v51/1980-220X-reeusp-51-e03248.pdf

DOI: 10.1590/S1980-220X2016046703248.

Cunha, J. A. (2001). Escalas Beck-manual [Beck Scales - manual]. São Paulo, SP: Casa do Psicólogo.

Egbe C. O., Dakum P. S., Ekong E., Kohrt B. A., Minto J. G., \& Ticao C. J. (2017). Depression, suicidality, and alcohol use disorder among people living with HIV/AIDS in Nigeria. BMC Public Health, 17. DOI:10.1186/s12889-017-4467-5

Evans D. L., Leserman J., Perkins D. O., Stern R. A., Murphy C., \& Zheng B. (1997). Severe life stress as a predictor of early disease progression in HIV infection. Am J Psychiatry 
154(5). Recuperado de https://ajp.psychiatryonline.org/DOI/abs/10.1176/ajp.154.5.630 DOI: 10.1176/ajp.154.5.630

Fernández M. D. S, Torvisco D. T. (2014). Síndromes psiquiátricos en personas infectadas con el Virus de la Inmunodeficiencia Humana: una revisión breve. Ver Neuropsiquiatr, 77(2), 70-77.

Gandini R. C., Martins M. C. F., Ribeiro M. P. \& Santos D. T. G. (2007). Inventário de Depressão de Beck - BDI: validação fatorial para mulheres com câncer. Psico-USF, 12(1), 23-31.

Gaspar J. G., Reis R. K., Pereira F. M. V., Neves L. A. S., Castrighine C. C., \& Gir E. (2011). Quality of life in women with HIV/AIDS in a municipality in the State of São Paulo. Rev Esc Enferm USP, 45(1), 235-31.

Goldberg D. P., Cooper B., Eastwood M. R., Kedward H. B. , \& Shepherd M. A. (1970). Standardized psychiatric interview for use in community surveys. Br J Prev Soc Med, 24,18-23. Recuperado de https://www.ncbi.nlm.nih.gov/pmc/articles/PMC1059220/

Junior P. R. B. S., Szwarcwald C. L., \& Castilho E. A. (2011). Self-rated health by HIV-infected individuals undergoing antiretroviral therapy in Brazil. Cad. Saúde Pública, 27. DOI: 10.1590/S0102-311X2011001300007.

Junqueira P., Bellucci S., Rossini S., \& Reimão R. (2008). Women living with HIV /AIDS. Arq Neuropsiquiatr, 66(4), 817-820.

Liu Y., Niu L., Wang M., Chen X., Xiao S \& Luo D. (2017). Suicidal behaviors among newly diagnosed people living with HIV in Changsha, China. AIDS Care, 29(11), 1359-1363. Recuperado de https://www.ncbi.nlm.nih.gov/pubmed/28593797. DOI: 10.1080/09540121.2017.1338653

Lopes N. L. V., Gracitelli C. P. B., \& Moura C. R. (2018). Creation of a childhood glaucoma registry database. Arq Bras Oftalmol, 81(4), 271-5. Arq Bras Oftalmol. 2018;81(4):271-5. DOI: $10.5935 / 0004-2749.20180055$

Marcolino J. A. M., \& Iacoponi E. (2003). The early impact of therapeutic alliance in brief psychodynamic psychotherapy. Rev bras psiquiatr, 25(2), 78-86.

Mari, J. J., \& Williams, P. (1986). A validity study of a psychiatric screening questionnaire (SRQ-20) in primary care in the city of São Paulo. British Journal of Psychiatry, 148(1), 23-26. Recuperado de https://www.ncbi.nlm.nih.gov/pubmed/3955316. DOI:10.1192/bjp.148.1.23

Mola C. L., Horta B. L., Gonçalves H., Quevedo L. A., Pinheiro R., Gigante D. P., . . Barros F. C. (2016). Breastfeeding and mental health in adulthood: A birth cohort study in Brazil. Journal of Affective Disorders, 202, 115-119. DOI: 10.1016/j.jad.2016.05.055.

Morais L. C., Zanuto E. A. C., Queiroz D. C., Yndawe M., Araújo C., Rocha A. P. R., . . . Codogno J. S. (2017). Association between sleep disorders and chronic diseases in patients 
of the brazilian national health system. J. Phys Educ, 28. DOI: 10.4025/jphyseduc.v28i1.2844.

Mugusi S., Ngaimisi E., Janabi M., Mugusi F., Minzi O., Aris E., . . . Aklillu E. (2018). Neuropsychiatric manifestations among HIV-1 infected African patients receiving efavirenz-based cART with or without tuberculosis treatment containing rifampicin. Eur $J$ Clin Pharmacol. Recuperado de https://www.ncbi.nlm.nih.gov/pubmed/30003275 DOI: 10.1007/s00228-018-2499-0

Naciones Unidas. (2015). Programa conjunto de las Naciones Unidas sobre el VIH/Sida. Ginebra: $\quad$ Onusida. Recuperado de http://www.unaids.org/sites/default/files/media_asset/AIDS_by the_numbers_2015_es.p $\underline{\mathrm{df}}$

Nakimuli-Mpungu E, Mojtabai R., Alexandre P. K., Katabira E., Musisi S., Nachega J. B., Bass, J. K. Cross-cultural adaptation and validation of the self-reporting questionnaire among HIV+ individuals in a rural ART program in southern Uganda (2012). HIV/AIDS - Research and Palliative Care, 4. Recuperado de https://www.ncbi.nlm.nih.gov/pubmed/22570575 DOI: 10.2147/HIV.S29818

Passos S. M. K., \& Souza L. D. M. (2015). An evaluation of quality of life and its determinants among people living with HIV/AIDS from Southern Brazil. Cad. Saúde Pública, 31(4), 800-814. DOI: 10.1590/0102-311X00000514

Rachid M. \& Schechter M. Manual de HIV/AIDS (2008). $9^{\text {a }}$ Edição Revista e Ampliada. Editora Revinter.

Reis R. K., Castrighini C. C., Melo E. S., Jesus J. G., Queiroz A. A. F. L., \& Gir E. (2017). Avaliação dos sintomas depressivos somáticos e afetivo-cognitivos de pessoas vivendo com HIV/AIDS. Acta Paul Enferm, 30(1), 60-5. DOI: 10.1590/1982-0194201700009

Salle E., Rocha N. S., Rocha T. S., Nunes C., \& Chaves M. L. F. (2011) Escalas psicométricas como instrumentos de rastreamento para depressão em estudantes do ensino médio. Psiq Clin, 39(1), 24-7.

Schlebusch L., \& Govender R. D. (2015). Elevated Risk of Suicidal Ideation in HIV-Positive Persons. Depress Res Treat. DOI: 10.1155/2015/609172

Sin N. L., \& DiMatteo M. R. (2014). Depression Treatment Enhances Adherence to Antiretroviral Therapy: A Meta-Analysis. Ann Behav Med, 47(3), 259-269. DOI:10.1007/s12160-013-9559-6

Tancredi M. V., \& Waldman E. A. (2014). Survival of AIDS patients in Sao Paulo-Brazil in the pre-and post-HAART eras: a cohort study. BMC Infect Dis. 14(1),1-8. Recuperda de https://www.ncbi.nlm.nih.gov/pmc/articles/PMC4247874/. DOI: 10.1186/s12879-0140599-8

Treisman G. J., \& Soudry O. (2016). Neuropsychiatric effects of hiv antiviral medications. Drug Saf, 39(10), 945-57. Recuperado de https://www.ncbi.nlm.nih.gov/pubmed/27534750. DOI: 10.1007/s40264-016-0440-y. 
Vitoria M., Hill A., Ford N., Doherty M., Clayden P, Venter F., . ., Domanico P. L. (2018). The transition to dolutegravir and other new antiretrovirals in low-income and middleincome countries: what are the issues? AIDS, 32(12), 1551-1561. Recuperado de https://www.ncbi.nlm.nih.gov/pubmed/29746295.

DOI: 10.1097/QAD.0000000000001845

Wang W., Xiao C., Yao X., Yang Y., Yan H., \& Li S. (2018). Psychosocial health and suicidal ideation among people living with HIV/AIDS: A crosssectional study in Nanjing, China. Plos One, 13(2). DOI:10.1371/journal.pone.0192940

\section{Os Autores:}

Aurélio Goulart Rodovalho é graduado em Biomedicina pela Pontifícia Universidade Católica de Goías. especialista em Gestão Hospitalar e Serviços de Saúde (UFG), mestre em Gestão Organizacional (UFG). Atualmente Coordenador do Centro Municipal de Diagnósticos, Coordenador do Centro de Testagem e Aconselhamento (CTA), Coordenador do Serviço de Atendimento Especializado (SAE) e Coordenador Municipal de DST-Aids e Hepatites virais de Catalão/GO, e-mail: aureliorodovalho@ hotmail.com

Roselma Lucchese é Enfermeira. Doutora em Enfermagem/EEUSP. Docente no programa de Pós-Graduação em Gestão Organizacional da Universidade Federal de Goiás. Prof. titular na UFG - Regional Catalão. E.mail: roselmalucchese@hotmail.com

Moisés Fernandes Lemos possui Licenciatura em Psicologia (1986), Formação de Psicólogo (1987) e Especialização em Filosofia (1990), pela Universidade Federal de Uberlândia, Especialização em Psicologia Clínica, pelo Conselho Federal de Psicologia (2001), Mestrado em Psicologia pela Universidade Federal de Uberlândia (2006), Doutorado em Educação, pela Universidade Federal de Goiás (2014), Pós-doutorado em Saúde Coletiva, pela UNICAMP (2017/2018). Atualmente é professor adjunto da Universidade Federal de Goiás (Regional Catalão), e-mail: moisesflemos@ yahoo.com.br

Henrique Senna Diniz-Pinto possui graduação em Engenharia de Minas pela Universidade Federal de Ouro Preto (1989), doutorado em Mineralogia na Universität Johannes Gutenberg Mainz (1999) e pós-doutorado em Mineralogia e Caracterização Mineral na Universidade de Brasília (2000-2003) como bolsista-recém doutor. Tem experiência em Geociências, na área de Mineralogia, Cristalografia, Cristaloquímica, Mineralogia Analítica e Aplicada e Caracterização Mineral. E-mail: hsennadiniz@terra.com.br

Lucas Barros Araújo é acadêmico do curso de graduação em Enfermagem pela Universidade Federal de Goiás/ Regional Catalão. Colaborador no Grupo de Pesquisa Interdisciplinaridade em Ciências da Saúde (INCISA). Bolsista do Programa Institucional de Bolsas de Iniciação Científica (PIBIC - CNPq). E-mail: lucas.enf8@gmail.com

Patrícia Mendes Barros é acadêmica do curso de graduação em Enfermagem pela Universidade Federal de Goiás/ Regional Catalão. Colaboradora no Grupo de Pesquisa Interdisciplinaridade em Ciências da Saúde (INCISA). Bolsista do Programa Institucional de Bolsas de Iniciação Científica (PIBIC - CNPq). E-mail: patricia.mbarros2@gmail.com

Recebido em: 21/08/2018.

Aprovado em: 14/10/2018 